\title{
Possibility of Constructive Surface Plasmon Scattering in Organic Light Emitters
}

\author{
Y. Mizoguchi ${ }^{1}$, T. Yoneda ${ }^{1}$, T. Inui ${ }^{1}$, T. Ishiguro ${ }^{1}$, K. Kasahara ${ }^{1}$, N. Ikeda ${ }^{2}$, Y. Sugimoto $^{2}$ \\ ${ }^{1}$ Graduate School of Science and Engineering, Ritsumeikan University, \\ 1-1-1 Noji-higashi, Kusatsu, Shiga 525-8577, Japan \\ kasahara@se.ritsumei.ac.jp \\ ${ }^{2}$ Nanotechnology Innovation Center, National Institute for Materials Science (NIMS), \\ 1-2-1 Sengen, Tsukuba, 305-0047, Japan
}

\section{Extended Abstract}

It is of scientific and practical significance to study surface plasmon polariton (SPP) scattering-enhanced light emission which occurs in organic light emitting diodes. Energy transfer from nonradiative components in an organic layer to SPPs could be extracted as light through the SPP scattering at submicrometer-scale roughness on the surface of a metal film, which affects the light-emitting efficiency of the organic layer. So far, there have been some experimental investigations on this using low molecular weight organic materials and polymers. PL enhancement reached, for example, $\sim 10$ in the normal direction with $\mathrm{Alq}_{3}$ on $\mathrm{Au}$, compared with $\mathrm{Alq}_{3}$ without $\mathrm{Au}$ [1-3]. On the other hand, a rigorous formulation for the scattering of SPP from a one-dimensional surface irregularity was developed by the use of an impedance boundary condition, yielding an electromagnetic field above a metal [4]. Using the formula, one can obtain the scattering probability $\eta\left(=P_{s d} / P_{\text {inc }}\right)$, where $P_{s c}$ : total power carried away from a metal surface by SPP scattering, and $P_{\text {inc }}$ : power carried by an incident SPP. However, we found that numerical calculations could not account for the measured PL enhancement. One SPP is usually understood to be created by energy transfer from a neighboring exciton in the organic layer. This paper describes the possibility that multiple SPPs are generated in phase, leading to constructive interference occurrence among the scatted electromagnetic waves. The distance between the closest organic molecules is an order of $\mathrm{nm}$, and it is possible that SPPs are generated simultaneously rather than separately.

To confirm the SPP-enhanced PL, we measured the quantum efficiencies of $\mathrm{Alq}_{3} / \mathrm{GaAs}$ and $\mathrm{Alq}{ }_{3} / \mathrm{Ag} / \mathrm{GaAs}$ using an integrating sphere. The thickness of $\mathrm{Alq}_{3}$ was $60 \mathrm{~nm}$ for two samples, and $\mathrm{Ag}$ was $200-\mathrm{nm}$ thick. It was necessary to take into account the fact that the light collected with the $\mathrm{Alq}_{3} / \mathrm{Ag} / \mathrm{GaAs}$ sample was almost double. As a result, the quantum efficiency of $\mathrm{Alq}_{3} / \mathrm{Ag} / \mathrm{GaAs}$ was confirmed to be $\sim 2.5$ times higher than that of $\mathrm{Alq}_{3} / \mathrm{GaAs}$. Using a simple approach, $\mathrm{PL}$ enhancement is expressed as $\left(p-(1-\eta) p_{s}+(1-p) \zeta \eta\right) / p$. Here, $p$ is the radiative probability of an exciton without metal, $p_{s}$ is the SPP generation probability, and $\zeta$ is the energy transfer probability of the nonradiative component to SPP. We performed numerical calculations on $\eta$. The average heights of the bump and pitch measured by AFM were $7 \mathrm{~nm}$ and $100 \mathrm{~nm}$, and these averages were used in the calculation. The colliding times of SPP which experienced bumps was determined by the propagation distance of the SPP attenuating to 1/e due to ohmic loss. As a result, $\eta$ was found to be as small as 0.04 . If this is true, one can never explain the enhanced PL. The total energy that original $N$ excitons possess is, of course, proportional to $N$, regardless of individual phase of generated SPPs, whose electric field is expressed as $\sum_{j} E_{j} e^{i \theta_{j}} . P_{s}$ is proportional to the square of this term, and thereby becomes $N^{2} E^{2}$, when the respective phase is the same, and $E_{j}$ is equal to $E$. Therefore, the actual scattering probability of one SPP should increase in proportion to $N$, and becomes $N \eta$.

\section{References}

[1] M. C. Tam, H. Su, K. S. Wong, X. Zhu, and H. S. Kwok, "Surface-plasmon-enhanced photoluminescence from metalcapped Alq 3 thin film," Appl. Phys. Lett., vol. 95, pp. 051503, 2009.

[2] T. D. Neal, K. Okamoto, and A. Scherer, "Surface plasmon enhanced emission from dye doped polymer layers," Opt. Express., vol. 13, no. 14, pp. 5522-5527, 2005.

[3] T. Inui, Y. Mizoguchi, T. Yoneda, H. Kawase, K. Kasahara, N. Ikeda, and Y. Sugimoto, "Factors Determining the Light 
Amplification Spectra in Surface Plasmon-Enhanced Organic Light Emitters," in Proceedings of META'15, New York City, NY, 2015, P40.

[4] J. A. Sanchez-Gil and A. A. Maradudin, "Near field and far field scattering of surface plasmon polaritons by onedimensional surface defects," Phys. Rev. B, vol. 60, no. 11, pp. 8359-8367, 1999. 